\title{
Array Pattern Synthesis Using Numerical Pattern Synthesis Algorithm Including Mutual Coupling
}

\author{
Shu-Lin Chen, Pei-Yuan Qin, Y. Jay Guo \\ Global Big Data Technologies Centre, \\ University of Technology Sydney (UTS), \\ Sydney, NSW, Australia \\ ShulinChen.UTS@gmail.com
}

\author{
Yanhui Liu, and Pengfei You \\ Department of Electronic Science, \\ Xiamen University, \\ Xiamen, Fujian, China \\ yanhuiliu@xmu.edu.cn
}

\begin{abstract}
In this paper, numerical pattern synthesis (NPS) algorithm integrated with seeded region growing (SRG) method is proposed to synthesize the array in the presence of mutual coupling. In order to obtain the mutual coupling between the array elements, active pattern technique is used to find active element patterns. A $4 \times 8$ microstrip antenna array is employed to validate the effectiveness of the proposed algorithm including mutual coupling.
\end{abstract}

\section{INTRODUCTION}

Numerical pattern synthesis (NPS) algorithm is a classical array synthesis method based on the adaptive array theory, systematically proposed by Olen and Compton in [1]. The basic idea is that, by checking the difference between the current pattern and the desired pattern, numbers of discrete artificial interferences are assigned in the sidelobe region to achieve the desired sidelobe performance. During this process, the sidelobe region should be precisely discriminated from the array pattern in order to place the interferences in the sidelobe region rather than the mainlobe region.

Based on the above classical NPS algorithm, by minimizing the weighted norm of the difference between the desired pattern and the current pattern using the weight function, which is similar to the artificial interferences used in NPS, the shaped pattern can be obtained in [2]. In [3], Li, et al., applied amended amplitude approaching algorithm to improve the convergence rate. Despite of the improvements, one important issue has not been discussed in the previous work, that is in each iteration, the mainlobe region changes with the sidelobe level, and should be determined accurately to place the interferences appropriately.

Regarding the problem of determining the mainlobe region of an arbitrary pattern, few methods such as the linear search method and the central difference method have been reported, and they can be only applied to deal with the case of onedimensional patterns in the linear array. In this paper, a seeded region growing (SRG) method, which is used for the image segmentation in image processing, is employed to determine the mainlobe region of an arbitrary pattern. Based on this mainlobe determination process, NPS algorithm integrated with SRG method is straightly applied in the array synthesis. Moreover, to consider the array mutual coupling, the active element pattern technology in [4] is used to obtain the element patterns.

The organization of this paper is shown as follows. Section II gives the algorithm description of the NPS algorithm integrated with SRG method including mutual coupling. Section III presents a numerical example. A conclusion is made in Section IV.

\section{Algorithm DESCRIPTION}

The aim of this array synthesis is to find optimum weights that can enable the array to achieve desired sidelobe performance with a focused beam at point $\left(\theta_{d}, \phi_{d}\right)$. NPS algorithm integrated with SRG method considering the mutual coupling is briefly described in the following:

For an arbitrary N-element array, the array steering factor $\mathbf{a}(\theta, \phi)$ can be calculated by

$$
\mathbf{a}(\theta, \phi)=\left[a_{1}(\theta, \phi), a_{2}(\theta, \phi), \cdots, a_{N}(\theta, \phi)\right]^{T}
$$

where $a_{n}(\theta, \phi)=f_{n}(\theta, \phi) e^{-j \psi_{n}(\theta, \phi)}$, and $j=\sqrt{-1} . f_{n}(\theta, \phi)$ is $n$th element scalar pattern. $\psi_{n}(\theta, \phi)=\frac{2 \pi}{\lambda}\left(x_{n} \sin \theta \cos \phi+\right.$ $y_{n} \sin \theta \sin \phi$ ) is the $n$th element phase difference in terms of the array phase center. And the far-field array pattern is

$$
G(\theta, \phi)=\left|\mathbf{w}^{H} \mathbf{a}(\theta, \phi)\right|
$$

The weights can be obtained by minimizing the interferences and noise (SINR) powers while keeping the desired angle power to be maximum. Using the minimum variance distortionless response (MVDR) beamforming approach, weight vector $W(k)$ can be expressed as

$$
W(k)=\frac{[\mathbf{R}(k)]^{-1} \mathbf{a}\left(\theta_{d}, \phi_{d}\right)}{\mathbf{a}^{H}\left(\theta_{d}, \phi_{d}\right)[\mathbf{R}(k)]^{-1} \mathbf{a}\left(\theta_{d}, \phi_{d}\right)}
$$

Where $\mathrm{k}$ is the number of current iterations, and $\mathbf{R}(k)$ is the array covariance at $k$ th iteration.

In each iteration, the mainlobe region is increased as the sidelobe level reduces. Here, SRG method is proposed to accurately and robustly determine the mainlobe region. The main idea of SRG method is to start with a set of seed points, and then append to each seed from these grow regions with the neighboring pixels that have properties similar to the seed [5], [6] as shown in Fig. 1. As can be seen, the seed can grow in four directions, that is, $\{(-1,0),(1,0),(0,-1),(0,1)\}$. The 
growing procedure will not stop until the seeds can not grow any more.

Regarding the mutual coupling issue, the element patterns are not assumed to be the same or have specific expressions. They are obtained from the real array environment, that is the active element pattern. In this way, as is seen in Equation (1), the steering vector is calculated using these active element patterns, that is, $f_{n}(\theta, \phi)$ uses the active scalar pattern of the $n$th element. Thus, the excitation weights are get including the mutual coupling in the array synthesis. Integrated with the SRG method, NPS algorithm in the presence of mutual coupling is utilized to synthesize the array with arbitrary pattern shapes of the mainlobe region.

\begin{tabular}{|l|c|c|}
\hline Pixel(-1,-1) & Pixel(0,-1) & Pixel(1,-1) \\
\hline Pixel(-1,0) & Seed $(0,0)$ & Pixel(1,0) \\
\hline Pixel(-1,1) & Pixel(0,1) & Pixel(1,1) \\
\hline
\end{tabular}

Fig. 1. Seeded region growing.

\section{NUMERICAL EXAMPLE}

To include the mutual coupling in the synthesis, a $4 \times 8$ array with microstrip patch element antenna is synthesized. The array configuration is shown in Fig. 2. It has 4 arc arrays and each arc array has 8 elements. In this array, neighboring elements are all with $\lambda / 2$ space both in the azimuth and elevation planes. Each element has the same structure of conventional patch. The element pattern is obtained using the active element pattern technique, that is the element pattern is obtained in the array environment where other elements are all terminated with matched load. A HFSS simulation is set up based on the configuration shown in Fig 2. The desired goal is to achieve uniform $-30 \mathrm{~dB}$ sidelobe level pattern with a broadside beam. By following the process in Section II, this array is synthesized using the proposed NPS algorithm integrated with SRG method in the presence of mutual coupling. The optimized patterns are shown in Fig. 3. As can be seen, the pattern can exactly reach $-30 \mathrm{db}$ sidelobe with 50 iterations.

\section{CONCLUSION}

By integrating the SRG method to determine the mainlobe region, NPS algorithm is extended to synthesize the array with the focused patterns with arbitrary mainlobe shapes. Moreover, active element patterns are obtained using the active pattern technology, and thus the mutual coupling is included in the synthesis. A cylindrical array with microstrip antenna element is presented to validate the effectiveness of the NPS algorithm integrated with SRG method. Based on this, polarization synthesis could be conducted in our future work.

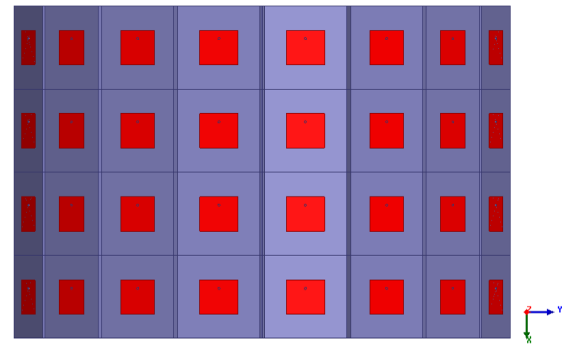

Fig. 2. Geometry of a $8 \times 4$ cylindrical array with the microstrip antenna element.

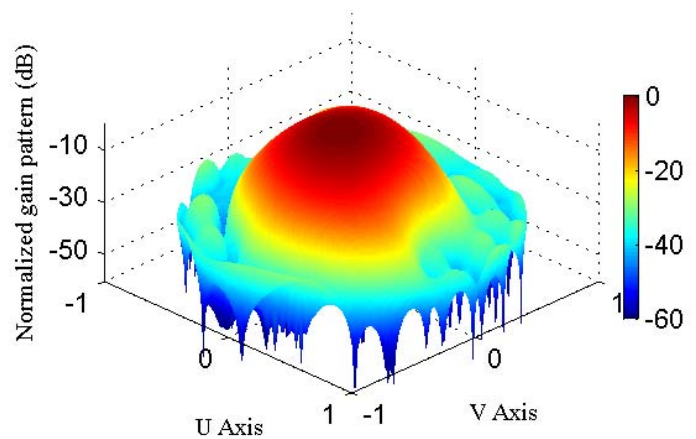

Fig. 3. The synthesized pattern by the proposed algorithm with 50 iterations for the $4 \times 8$ cylindrical array.

\section{REFERENCES}

[1] C. A. Olen and R. T. Compton, "A numerical pattern synthesis algorithm for arrays," Trans. Antennas Propag., vol. 38, no. 10, pp. 1666-1676, Oct. 1990.

[2] P. Y. Zhou and M. A. Ingram, "Pattern synthesis for arbitrary arrays using an adaptive array method," IEEE Trans. Antennas Propag., vol. 47, no. 5, pp. 862-869, May 1999.

[3] J. Li, R. Jin and Y. Sheng, "A fast synthesis algorithm of adaptive beams for smart antennas," Microw. Opt. Technol. Lett., vol. 36, no. 6, pp. 503507, Mar. 2003.

[4] D. M. Pozar, "The active element pattern," IEEE Trans. Antennas Propag., vol. 42, no. 8, pp. 1176-1178, Aug. 1994.

[5] R. Adams and L. Bischof, "Seeded region growing," Trans. Pattern Anal. Mach. Intell., vol. 16, no. 6, pp. 641-647, Jun. 1994.

[6] Y. Liu, S.-L. Chen, L. Zhang, and Q. H. Liu, "Determining the firstnull mainlobe region of an arbitrary pattern for 2-D numerical pattern synthesis algorithm," IEEE Trans. Antennas Propag., vol. 64, no. 3, pp. 1130-1136, Mar. 2016 